\title{
Carnets
}

Revue électronique d'études françaises de l'APEF

Première Série - 5 | 2013

Métamorphoses littéraires

\section{La métamorphose se mise a l'essai: Attraper ou miner le réel?}

\section{Zsuzsa Simonffy}

\section{(2) OpenEdition}

1 Journals

\section{Édition électronique}

URL : http://journals.openedition.org/carnets/8451

DOI : 10.4000/carnets.8451

ISSN : 1646-7698

Éditeur

APEF

\section{Édition imprimée}

Date de publication : 1 mai 2013

Pagination : 173-192

Référence électronique

Zsuzsa Simonffy, "La métamorphose se mise a l'essai: Attraper ou miner le réel? », Carnets [En ligne], Première Série - 5 | 2013, mis en ligne le 23 juin 2018, consulté le 19 avril 2019. URL : http:// journals.openedition.org/carnets/8451; DOI : 10.4000/carnets.8451

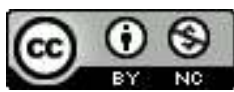

Carnets est mis à disposition selon les termes de la licence Creative Commons - Atribution - Pas d'utilisation commerciale 4.0 International. 


\title{
LA MÉTAMORPHOSE MISE À L'ESSAI \\ Attraper ou miner le réel?
}

ZSUZSA SIMONFFY

Université de Pécs

ffy.zsu@gmail.com

\begin{abstract}
Résumé
Pour donner sens aux relations que le sujet entretient avec l'univers d'objets il y a deux voies que le narrateur peut suivre dans son récit: la voie mimétique ou la voie fantastique. Ce travail a pour objectif d'explorer le processus métamorphique en s'interrogeant sur les modalités de la mise en discours du changement dans quelques récits dits fantastiques. Nous allons nous intéresser à la question de savoir comment les récits révèlent le spectacle d'une métamorphose, comment ils articulent les étapes du changement physique ou mental, comment ils fournissent les indices qui permettent de les qualifier de métamorphiques, et à quels moments interviennent les procédures descriptives déshumanisantes chez deux auteurs, notamment Maurice Carême et Marcel Thiry. L'enjeu de l'analyse consiste à repenser les rapports entre la fiction que les œuvres exposent et le réel auquel elles renvoient.
\end{abstract}

\begin{abstract}
There are two ways a narrator can convey the subject's relationship with the world of objects: the mimetic or the fantastic. This work aims to explore the metamorphic process by examining the modalities of how change is portrayed in some fantasy stories. We will look at how these stories describe the process of metamorphosis, how they express the physical or mental phases of change, in what way they qualify as metamorphic and we examine in which instances authors turn to dehumanizing descriptions, especially two authors, Maurice Carême and Marcel Thiry. The goal of the analysis is to revisit the relationship between fiction their work depict and the reality to which they refer.
\end{abstract}

Mots-clés: espace, énigme, fantastique, littérature belge d'expression française

Keywords: space, enigme, fantastic, Belgian French-speaking literature 
le regard du créateur (le poète) se nourrit de sa propre énigme et que l'énigme, à son tour, suscite d'autres élans

Jean Orizet

et seulement pourraient-ils voir la métamorphose profonde qui s'opère en toute chose quand jaillit l'étincelle $d u$ renouveau

Marie Gevers

\section{Introduction}

Nos réflexions s'inscrivent dans le cadre général d'une problématique qui se ramène au sein des études littéraires à certaines tendances tout particulièrement motivées d'une part, par la remise en question de la fonction représentationnelle du récit - au sens aristotélicien du terme - et, d'autre part, par la mise en cause de l'approche unitaire de l'individu, indissociable de la catégorie de la totalité. En effet, depuis un certain temps, de nombreux travaux insistent sur le phénomène de l'éclatement qu'il soit rapporté au sujet social impliquant l'interculturel (Lipovetsky, 1985), au sujet parlant impliquant le dialogisme et la polyphonie des énonciateurs dans la construction du sens par et dans le discours (Ducrot et Schaeffer, 1995), ou encore aux genres littéraires impliquant l'hétérogénéité et l'hybridité (Dambre et Gosselin-Noat, 2001).

Depuis les grands romanciers du $X^{e}$ siècle, Proust, Camus, Robbe-Grillet, entre autres, choisissant chacun une stratégie qui remet en question la fonction représentationnelle du récit en vue de le libérer du critère de cohérence interne à l'agencement des actions de l'intrigue, on peut repérer une tendance à rendre la visée autotélique de plus en plus patente sur la base du glissement opéré de l'univers fictif vers le discours romanesque. Mais comment en rendre compte?

De nos jours, dans les milieux académiques, il est devenu clair que la recherche de nouveaux outils d'analyse sera désormais indissociable de la construction même de l'objet d'étude relatif à toute discipline concernée. II en est de même sur le plan des études littéraires. Ce qui n'est pas sans rappeler qu'aucun consensus ne se livre, à ce jour, sur l'objet qui pourrait être délimité en tant que littéraire. Pour s'orienter dans ce champ ouvert par le caractère indéterminé de l'objet, d'un côté, et, par une multiplicité de méthodes et de théories, de l'autre, une des possibilités revient à avoir recours à des principes heuristiques susceptibles de guider la réflexion sur les aspects divers du phénomène considéré comme littéraire.

Notre travail consistera à établir et à mettre en œuvre un principe heuristique, plus précisément à montrer comment constituer un outil par le biais duquel nous avons accès à 
un objet d'étude, ledit éclatement impliquant des êtres multiples, fragmentés et contradictoires. Nous attribuerons donc le statut d'outil méthodologique à la métamorphose à travers laquelle il nous sera possible d'observer la mise en scène discursive du sujet en procès, incarnant une identité instable.

II s'agit là des premiers éléments d'un vaste projet au sein duquel notre interrogation portera sur les dispositifs textuels par lesquels les œuvres soumises à notre analyse rendent compte du changement, et sur les stratégies d'écritures par lesquelles est inscrit l'intérêt tout particulièrement pour la métamorphose. Nous avons repéré, dans notre corpus constitué des récits signés par des écrivains de la littérature belge d'expression française ${ }^{1}$ une stratégie narratologique qui consiste à prendre une même attitude envers le réel et l'imaginaire. Cette stratégie correspond, dans une perspective différente de la nôtre, à ce que Augé (1999) appelle monde contemporain au sein duquel coexistent des mondes divers relatifs à un même espace-temps.

Dans la perspective formelle de la poétique moderne, nous proposerons de dégager quelques éléments de ce corpus que la métamorphose permet de rendre homogène malgré toute disparité qu'on peut observer dans les modalités d'écriture et dans les manifestations diverses de la représentation battue en brèche. Dans le cadre de cette étude, nous nous appuyerons sur deux récits²: Médua (1976) de Maurice Carême et Nondum jam non (1966) de Marcel Thiry. Puisque ces récits sont considérés, dans les discours critiques, comme fantastiques (Lascu-Pop, 1994; Hallin-Bertin, 1981), quelques remarques préalables s'imposent à cet égard avant d'entreprendre l'analyse proprement dite.

\section{Fantastique: genre ou effet?}

Le fantastique est une catégorie incontournable en matière de la littérature belge, catégorie par rapport à laquelle sont fondées - explicitement ou implicitement - la plupart des approches visant à rendre compte de l'émergence de cette littérature. Comme le remarque Baronian qui insiste sur le caractère non conformiste de cette littérature:

L'école belge de l'étrange [...] conditionne tout le fait littéraire belge, à telle enseigne que ce domaine est sans doute le seul avec le surréalisme à travers lequel la

\footnotetext{
${ }^{1}$ Pour mener à bien ce projet, l'auteur a bénéficié d'une bourse de recherche accordée par les Relations Internationales Wallonie-Bruxelles, qui lui a permis de fréquenter avec assiduité les Archives et Musée de la Littérature.

${ }^{2}$ Le corpus contient également des œuvres suivantes: Anne Duguël (1998). Entre chien et louve. Paris: Denoël; Odilon-Jean Perier (1926). Le passage des anges. Paris: Gallimard - Nouvelle revue française; Paul Willems (1963). Il pleut dans ma maison. Bruxelles: Brepols; Thomas Owen (1972). La Truie et autres histoires secrètes. Verviers: Marabout; Éric de Haulleville (1936). Le voyage aux îles Galapagos. Paris: Bernard Grasset; Gabriel Deblander (1970). Le retour des chasseurs. Paris: Robert Laffont; Marie Gevers (s. d.). Nouvelles métamorphoses. Manuscrit.
} 
Belgique francophone peut se vanter de posséder une littérature vivante et dynamique. [...] C'est dire surtout qu'il existe chez les écrivains belges une disposition naturelle au fantastique (Baronian, 2000: 221).

Sans prétendre contester ou justifier cette disposition naturelle, il nous conviendrait d'envisager le fait que nombreux sont ceux ${ }^{3}$ qui considèrent le fantastique comme un genre autonome, apte à fonctionner sur la base de critères spécifiques suggérant ainsi deux acceptions bien distinctes, celle qui renvoie à l'imaginaire et par conséquent à la capacité de fantasmer, et celle qui évoque le surnaturel et donc l'inexplicable.

Nous ne reviendrons pas en détail sur une définition du fantastique par ailleurs largement étudié dans la critique et la théorie littéraires inspirées dans la plupart des cas des idées de Todorov, notre objectif n'étant pas de revendiquer une définition améliorée qui serait fondée de même sur des traits caractéristiques considérés comme conditions nécessaires et suffisantes. II est à noter que les genres commencent à devenir pertinents à partir du moment où ils sont repérables sur des frontières de catégories. Si elles sont a priori floues c'est parce qu'elles ne reposent pas sur les clivages de la différence et de l'identité responsables de stabilisations provisoires mais sur un principe de similarité assurant une dynamique intrinsèque.

Nous nous bornerons à situer la problématique générique à sa juste place en faisant remarquer, d'une part, qu'un genre, résultat d'une démarche déductive doit son existence à un ensemble de critères de définitions, et, d'autre part, que la démarche inductive n'est pas moins légitime en vue d'établir un genre historique sur la base d'un corpus d'œuvres, et dans le cas qui nous intéresse, permettant de construire ce qui fait le fantastique à telle ou telle époque ${ }^{4}$.

Or, en raison de la complexité même de ces questions on rencontre souvent, dans les discours critiques, une confusion entre ces deux démarches qui devraient pourtant produire - de manière disjointe - ou bien un genre théorique ou bien un genre historique. L'enseignement à tirer des études génériques consiste précisément à révéler le caractère disjoint des deux points de vue dont l'assimilation est à éviter dans un même cadre conceptuel dans la mesure où les catégories analytiques ne se laissent pas s'appliquer aux genres relevant d'une pratique d'écriture fondée sur une tradition culturelle, et les genres relatifs à une tradition culturelle ne sont accessibles autrement que par des catégories théoriques à tous ceux qui se situent à l'extérieur de cette tradition. Pour faire face à ce défi, il n'y a qu'une seule manière de procéder, à savoir, reconnaître que l'objet d'étude ne pourra pas concerner les genres usuels mais la logique d'une taxinomie qui les sous-tend, et en

\footnotetext{
${ }^{3}$ Pour une vision complète sur la question se reporter à Lits (1993: 8).

${ }^{4}$ Cette question est aussi traitée en terme d'architextualité dans la terminologie de Genette.
} 
assumer les conséquences. Dans la prise en compte des genres, les catégories analytiques interviennent nécessairement comme schémas transculturels et les "catégories" historiquement repérables fonctionnent plutôt sur la base de la ressemblance de famille.

S'il nous semble difficile, à partir des discours critiques que nous avons étudiés, de saisir la réalité que le terme fantastique subsume du fait qu'il a un contenu changeant, il n'est pas difficile d'enregistrer le fait que la simple considération générique à son propos lui attribue une reconnaissance institutionnelle. Sans contester donc cette reconnaissance institutionnelle ${ }^{5}$, retenons de ce que nous venons de décrire deux moments.

Premièrement, l'appartenance d'un texte donné à la catégorie fantastique n'est pas toujours jugée sur les mêmes critères. Ainsi, même si le terme fantastique désigne une classe de textes, les membres qui la constituent risquent de changer en fonction des critères choisis. De ce fait, il n'est pas étonnant que le fantastique suggérant l'impression du mystère soit appréhendé comme pouvant prendre des directions divergentes (Tonnet-Lacroix, 1993: 157) d'une part, vers les ressources traditionnelles telles que maléfices, diableries, sortilèges, etc., en somme, tout ce qui relève du surnaturel, et, d'autre part, vers l'intériorisation cherchant dans le monde la face cachée du réel. Sans parler du fait que faute de définition rigoureuse, la critique fait passer, souvent, au premier plan la variété du genre. Dans cet état de choses, on obtiendra par voie de sous-catégorisation les qualités spécifiques telles que merveilleux, magique, réel, etc., et ceci, pour être combinées entre elles.

Deuxièmement, le terme fantastique lorsqu'il n'est pas nettement d'ordre générique, désigne un comportement humain face à l'incertain, impliquant une logique qui échappe au rationnel, susceptible d'inclure ainsi mythes, légendes et magies. Comme l'a noté Marigny "le fantastique n'est pas un genre mais un mode de relation au monde" (Marigny, 1987: 20). Indépendamment du fait que le cadre dans lequel il se déploie soit réel ou imaginaire il est considéré comme phénomène littéraire produit des temps modernes et exclurait ainsi toute production orale. Cependant, dans le cas de figure où il n'est pas d'ordre générique mais d'ordre comportemental, il nous renvoie également, dans le domaine des recherches ethnologiques et anthropologiques, à des questions relatives à la dichotomie rationnel $v s$ irrationnel, ou à celles qui relèvent des pratiques sociales propres à des sociétés traditionnelles et à des sociétés occidentales.

Ainsi, pour nous, la question théorique de savoir s'il est possible ou pas d'en donner une définition uniquement sur la base des considérations formelles, se situe dans un autre registre que la question pratique des classements qui sont en circulation répondant à certains besoins, et dont on reconnaît les traces dans les mentions génériques à titre d'information sur les couvertures des livres publiés. Au lieu de suivre cette piste, nous

\footnotetext{
${ }^{5}$ Nous pensons ici à l'étiquette "littérature d'évasion".
} 
préférons avoir recours aux catégories narratives. Ce qui nous semble conforter leur pertinence conceptuelle est de pouvoir distinguer grâce à elles des techniques narratives mises en œuvre par des récits sans nécessité de désigner pour autant des classes de textes. Si nous parlons, malgré tout, de fantastique, pour nous, ce sera dans le sens d'effet qui peut être provoqué par les modalités de la narration aussi bien que par tout autre élément du récit ${ }^{6}$. Si plusieurs facteurs de tout ordre concourent à la production des effets fantastiques, cette diversité ne constitue pas a priori un obstacle pour identifier les textes comme étant membres de cette catégorie dont l'usage est cognitivement motivé. Nous nous hâtons d'y ajouter que cette reconnaissance ne nécessite pas la prise en compte des critères rigoureusement établis. Nous rappelons ce que Quaghebeur (2012) met en évidence par rapport au fantastique réel dans son étude sur Hellens, qui est donné en termes d'“effraction", "éphémère", "dimension d'éternité", "vide spatial", "hasard". Pour nous, indépendamment du fait que ces éléments soient des conditions nécessaires et suffisantes ou traits typiques, ils permettent en dernier ressort d'identifier et de reconnaître le fantastique dans tous ses effets.

\section{La métamorphose: état de l'art}

Si de nombreux travaux se préoccupent à circonscrire le domaine du fantastique, force est de reconnaître que jusqu'à présent la notion de métamorphose n'a pas été mobilisée de manière systématique en contexte du fantastique. Dans ce qui suit nous allons envisager les usages de la métamorphose dans les textes de la littérature belge convoqués plus haut. Avant de nous pencher sur les développements, il conviendrait de donner quelles sont les acceptions du concept pour pouvoir en sélectionner celles qui viendront à notre usage.

II va sans dire que le concept de métamorphose fixé par Ovide a connu, depuis, une fortune considérable traversant toutes les cultures de tous les temps ${ }^{7}$. Sans faire l'inventaire de toutes les formes de métamorphoses, il n'est peut-être pas inutile d'en rappeler quelques typologies qui sont en circulation.

Frontisi-Ducroux (2009) concentrée sur une période relativement restreinte de l'Antiquité propose une typologie à double fondement: d'une part, c'est l'origine donc intervention divine, et, d'autre part, c'est le résultat, à savoir l'apparence morphologique, de l'événement surnaturel qui l'emportent. Quant à l'intervention divine, elle implique à son tour deux types de transformation: ou bien ce sont les dieux qui la subissent prenant l'apparence

\footnotetext{
${ }^{6}$ Nous rappelons que Bouvet (1998) dégage parmi ces éléments l'ambiguïté, le suspense, le cadre de référence bousculé, les jeux de l'espace. Or, nous pouvons y objecter que toute ambiguïté ne provoque pas l'effet fantastique: il y en a qui provoque un effet humoristique.

${ }^{7}$ Pour une synthèse voir Brunel (2004).
} 
d'une entité autre, ou bien ce sont les êtres humains, les menaçant dans leur existence, qui en sont infligés. Quant à l'apparence morphologique, elle implique trois règnes respectivement d'ordre animal, végétal et minéral. Or, sur la base de cette typologie, la comparaison entre Ovide et les prédécesseurs grecs, ne risque d'apporter que des convergences. Par rapport aux Grecs qui pratiquent une écriture tendant à passer sous silence toutes les phases traversées, l'invention d'Ovide consiste à mettre en scène l'événement en question en décrivant les modifications phases par phases de telle manière que l'évolution manifeste une certaine continuité pour ainsi dire en rendant le dynamique. À l'origine, il s'agit donc dans le sens ovidéen du terme d'“un type de transformation qui échappe à l'ordre naturel et à l'action humaine" (Frontisi-Ducroux, 2009: 10), désignant d'un côté les mythes grecs qui racontent des changements, et d'un autre côté, visant la manière de la présentation en ralenti dans toutes ses phases que l'entité traverse. Par ailleurs selon le dictionnaire Robert, le mot métamorphose marque le passage sans transition d'un type d'organisation à un autre, ou encore le résultat de la modification. De toute manière, comme le rappelle Pigeaud: “L'une des difficultés les plus grandes, dans l'imagination de la métamorphose, [...] est de rendre le temps de la métamorphose, et de la montrer en acte" (Pigeaud, 1995: 116).

Par extension, le terme sorti du régime du mythe, de la fiction et de la poésie désignera plus tard d'autres catégories de changement telles qu'évolutions dans le sens zoologique ou migration des âmes et métempsychose indiquant une césure entre corps et esprit.

En élargissant le domaine des modèles mythiques proprement dit, Dubois (1985) met en œuvre des procédures permettant de catégoriser les différentes formes du changement. II en distingue cinq catégories qui sont à l'ordre: procédure de continuité censée rendre compte de l'idéologie du ralenti et du progrès continu, donnant lieu à la réforme; procédure de rupture censée rendre compte de l'idéologie de révoltes, inversions, révolutions; procédures de balancement et de retour représentant la rotation; procédure d'aveuglement censée rendre compte de fantasmes de l'ilinx et représentant l'idéologie du hasard; procédure de mort et renaissance représentant l'idéologie apocalyptique (Dubois, 1985: 17).

Les correspondances entre procédure et idéologies sont substituées dans l'analyse de Berthelot (1993) par un schéma qui a une valeur de référence. II montre par ailleurs une forte affinité avec le modèle actanciel ou au moins avec une grammaire du récit, du fait que les quatre paramètres ne sont pas sans rappeler la syntaxe de la phrase comme entité linguistique. Les voilà: le sujet qui la subit; l'agent qui la fait subir; le processus selon lequel elle s'accomplit; le produit qui en résulte, et auxquels nous nous hâterions d'ajouter un cinquième paramètre, le point de vue de celui qui prend en charge la métamorphose. 
Les différentes tendances que nous avons tenté d'esquisser ne sont pas exclusives les unes des autres et ne constituent pas des catégories qui seraient étanches. En effet, ces typologies semblent se recouvrir suivant l'étymologie du mot métamorphose composé du grec méta-: "qui dépasse" et du morphê: "la forme", désignant ainsi un mouvement général qui aboutit à un changement de forme. Les modifications peuvent affecter un être animé aussi bien qu'inanimé tantôt dans sa forme physique tantôt dans sa nature profonde.

Cependant, si elles ne se recouvrent qu'en partie c'est dans la mesure où le changement n'établit pas toujours une césure irréversible dans l'existence d'une entité qui, en conséquence des transformations subies, se sent devenir un autre. Le concept peut désigner ainsi non seulement un changement radical ou complet de l'entité mais aussi un travestissement. En ce qui concerne les transformations, il n'est peut-être pas inutile de distinguer la modification des apparences et la mutation profonde touchant plutôt au comportement des entités concernées.

Dans une perspective toute autre, à savoir d'ordre logico-sémantique, il sera possible de révéler qu'il y a effectivement deux mouvements en parallèle à envisager: la transformation implique déformation. Nous pouvons rendre compte de ce double caractère de la métamorphose en termes de reclassification et recatégorisation. Suivant les travaux d'Achard-Bayle (2001) intéressé à savoir quelles sont les traces linguistiques des changements laissées sur l'identité des entités, l'opération de reclassification permet de présenter une même entité sous divers aspects, sur laquelle pèse la contrainte d'une instance focale et narrative, alors que la recatégorisation est l'effet d'une évolution réelle. La particularité des récits vient précisément de la prédominance de la reclassification sur la recatégorisation, ou inversement.

Même si elle a été rapide et par conséquent incomplète, notre présentation a mis en évidence quelques points de repère dans les interrogations sur les modalités de la mise en discours du changement. Nous allons étudier un roman qui met en scène les phases successives du processus même, et un autre, qui, au contraire, les dissimule pour n'en rapporter que l'aboutissement.

\section{Les usages de la métamorphose: perspective visuelle}

Nous allons organiser nos propos autour du processus d'altération. Quant au roman de Maurice Carême, Médua nous émettons l'hypothèse selon laquelle le processus d'humanisation de la méduse que le narrateur ramasse à la plage pour la ramener chez lui doit être indissociable de son regard, alors que le processus d'animalisation de la femme que le narrateur croise et entrecroise - et appelle Médua -, situé en dehors du champ de vision de ce même regard doit être laissé sous-entendu. Cette dissymétrie ne permettant pas 
d'attribuer la transformation à deux sens à une seule et même entité impliquerait deux récits: celui de l'histoire d'une métamorphose et celui qui constitue une énigme dont l'objet serait la métamorphose présentée dans ses détails par l'autre récit. Notons que dans notre approche, ce caractère énigmatique contribue largement à susciter l'effet de fantastique qui ne vient donc pas de l'univers représenté - par ailleurs affecté de visions oniriques -, mais de ce genre d'agencement dépendant de la situation de l'énonciation de l'instance narrative.

Le récit ouvre sur une rencontre de trois personnages - une femme, son compagnon et le narrateur-témoin - que le hasard amène d'abord dans un même compartiment, et ensuite dans un même tram. Cette rencontre est racontée sur la base de la perception visuelle à savoir au travers du regard fasciné du narrateur-témoin, qui se fixe sur le visage de la femme "étonnamment rond et aplati", sur "le vert transparent de ses prunelles" et sur "son teint laiteux" (Carême, 1976: 11). Si cette description est pertinente en incipit, c'est parce que c'est l'apparence qui est directement accessible au narrateur en situation de voyage sans rompre réellement avec le souci de vraisemblance. Ce visage ne saurait se réduire cependant à une simple description de traits physiques, puisqu'il s'impose: il se fait marquer par une plaie produite lors d'un accident pour se faire remarquer encore davantage. La description loin d'être insignifiante anticipe sur l'intériorité du narrateur-écrivain, et par conséquent, sur le récit de métamorphose.

Quand les trois personnages se retrouvent quelque peu après dans le tram, le même regard bute encore sur des "signes étranges" qui marquent cette fois un bagage appartenant au couple. Il est à noter que le regard de la femme d'abord détourné dans le train sera ensuite dirigé dans le tram vers le dehors pour se faire déployer "la plaine flamande" peuplée "de tournesols" (Carême, 1976: 12). Cette exploration de l'espace est significative pour la symbolique des couleurs: la robe jaune et phosphorescente de la femme sera un motif récurrent.

Par ailleurs, si la perception auditive est également impliquée dans les scènes qui réunissent les trois personnages dans un même espace, c'est pour bloquer toute communication possible. Le narrateur-témoin insistant ainsi sur l'étrangeté du couple n'arrive à capter ni dans le compartiment ni dans le tram que des mots incompréhensibles. II croit entendre cependant dire Médua, et il est satisfait d'obtenir ainsi le nom de la femme mystérieuse. S'ils ne parlent pas le langage de celui qui les observe, cette amorce suggère que le narrateur-témoin doit révéler, dans ce qui suit, un mystère ou au moins déchiffrer un secret. Cependant ce n'est pas lui qui part à la recherche d'un mystère mais c'est le mystère qui ne cesse de le hanter et de l'obséder.

Suite à ces indices devenus saillants lors de la rencontre, le narrateur-témoin, sous l'effet de la fascination exercée par le curieux couple, aura quelque chose à raconter. C'est 
ainsi que se crée l'attente à l'égard d'un récit qui s'articulera désormais sur la réitération de leurs rencontres fortuites.

Le mode d'appréhension du monde pour le narrateur consiste en l'acuité du regard sur le monde impliquant une évolution métamorphique dans l'espace. En effet, l'espace clos du narrateur revêtant des attributs non habituels va s'humanisant sous l'apparence de la femme du train. Cet espace concret de la chambre de la villa dans laquelle le narrateurécrivain s'installe, est désormais habité de l'image de la femme qui ne cessera de le hanter. Le texte est centré sur les différents modes de réapparitions du visage de la femme dans le vide de l'intérieur de la maison aussi bien que dans le vide de l'extérieur. Le regard du narrateur-écrivain qui est dans un état engourdi sous la chaleur continue à capter des signes étranges dans son environnement tels que la "lumière rose", "les chemises roses", "la pièce teintée de rose" suggérant ainsi la prégnance de l'existence féminine.

C'est à partir de ce moment où les attributs physiques prennent déjà une détermination explicitement aquatique: "elle [la salle à manger] paraissait plongée dans un aquarium rempli de soleil [...] cet aquarium me rappelle la couleur des yeux de Médua" (Carême, 1976: 13). Ou plus loin "Je me tournai vers la mer. Elle avait la couleur des yeux de Médua" (Carême, 1976: 42). Cette détermination aquatique n'est pas le produit d'un jeu de hasard. Nous trouvons une remarque de Frontisi-Ducroux particulièrement pertinente dans ce contexte concernant les divinités marines: elles "sont polymorphes, à l'image sans doute de leur milieu naturel, l'élément aquatique" (Frontisi-Ducroux, 2009: 11). Le milieu naturel d'ordre aquatique rend pour la méduse la métamorphose constitutive de son être, ce qui est largement exploité dans la mise en discours.

Cet espace tend à s'ouvrir vers le dehors: la présence du monde extérieur s'impose dans la mesure où les menus détails sans aucun intérêt demandent à être enregistrés pour être visibles. Cet enregistrement donne accès à tout ce qui dépasse normalement les segments découpés par l'empan visuel du narrateur. À travers une image d'un espace qui échappe par ailleurs nécessairement à tout regard, le narrateur fait subir à l'espace ainsi étendu une métamorphose. Si ce dehors avec la plaine flamande, la mer d'un jaune vert, la plage encadre aussi son récit, c'est parce que les contours du paysage déformés donnent à voir de grands animaux allongés. Le moindre mouvement du monde à condition qu'il soit rapporté à ce narrateur rend du coup ce monde accessible sous son angle de vision.

Les attributs physiques de la femme qui marquent désormais l'environnement du narrateur correspondent absolument aux attributs de la méduse. En effet, ce sont les indices qui devraient anticiper le processus de l'animalisation. Or, c'est seulement dans le cas du processus de l'humanisation que le narrateur ressent le besoin de le mettre en scène. Nous pouvons formuler déjà l'énigme: Est-ce la femme qui présente une apparence animale avant de retrouver sa forme humaine? Est-ce la méduse qui présente une apparence humaine 
avant de retrouver sa forme animale? Ou tout simplement comment se fait-il que la femme soit méduse et la méduse soit femme?

Chaque disparition est suivie d'une réapparition. La rencontre se répète dans le temps d'abord avec Malbot, l'homme du train: "le ruban rose" sert à établir son identité et "la réclame suggestive" (Carême, 1976: 14) à confirmer en quelque sorte l'hypothèse du narrateur-témoin sur leur métier. S'il n'est pas artiste dans le sens strict du terme, il est illusionniste. Ensuite une autre rencontre avec Médua "au coin du grand café". L'acuité du regard commence à être non fiable: "Plus je la regardais, plus elle me semblait irréelle [...] Je m'avançai [...] Tout redevient affreusement réel" (Carême, 1976: 17). En ce qui concerne ce narrateur homodiégétique, il n'est pas anonyme, au contraire, il coïncide avec l'auteur. II se présente à la fois comme secondaire dans la mesure où l'héroïne c'est Médua et comme héros dans la mesure où c'est lui qui est accusé, tout à la fin, d'avoir tué Médua. Cette myopie semble garantir pour le narrateur-écrivain la distance nécessaire à être assuré du fait que chaque moment vécu n'existe que lorsqu'il est revécu: or, lorsque ces moments sont repensés grâce au travail de la mémoire dans l'acte d'écriture, ils ne fournissent rien que de l'incohérence.

La rencontre suivante a lieu à l'intérieur du café. Le narrateur assiste ici à la séance dans laquelle Malbot décapite Médua et pour laquelle le vol de deux papillons de nuit assure un décor insolite, mettant en cause l'acuité de son regard. L'effort du narrateur, afin de retrouver Médua dont le chant plaintif est le seul contact possible, met en jeu le motif de la quête. Or, plus il est incité à la recherche de Médua, plus il se retrouve dans l'incapacité d'écrire. Dans la progression induite par la quête, le geste métafictionnel fait intervenir une rupture. Le narrateur lors de la quête est à une grande proximité de ce qu'il observe, mais il semble en être éloigné dès qu'il se voue à tenter de mettre en mots ses observations.

En effet, les observations prennent la forme d'idées, de sentiments, d'images mais qui ne donnent guère un tout cohérent sur le papier faute de facteur organisateur. Ce qui a pour conséquence qu'il devient étranger à lui-même. À partir de ce moment, il a des hallucinations dont celle du "soldat assis sur le lit" est intensément visualisée. Dans ce contexte, la perception auditive est aussi évoquée sous formes de chant et musique. Une fois ses premières hallucinations disparues, sous l'effet des sonorités musicales qui continuent de résonner de l'intérieur, il retrouve de l'inspiration qui ne dure pas pourtant longtemps.

Lors de la quête, attiré par le chant lointain de Médua, le narrateur-témoin se retrouve à la plage où la voix humaine d'une des méduses le prie de la ramener chez lui. Sur la base d'une ressemblance initiale très forte avec une figure humaine, la fissure comme une bouche, la méduse se métamorphose sous le regard du narrateur-témoin. L'imagination occupe son esprit tout entier et il charge progressivement l'animal de toutes ses obsessions. 
L'être qui se transforme sous son regard n'effectuera pas cependant un passage qui aboutirait à revêtir définitivement une nouvelle forme, ce qui est motivé par une série de descriptions introduites par "comme" induisant ainsi le premier degré de la métamorphose.

Le narrateur-témoin met sous les yeux la métamorphose de manière à ce que la transformation d'animal en humain se produise d'étape en étape. Le processus de l'humanisation se donne à voir à travers l'adaptation de la méduse aux conditions de son nouveau milieu qui d'aquatique devient terrestre. Puisque la méduse mange la même nourriture que son patron, la première phase met en scène la manière dont "les bords de la fissure" deviennent "modelés en lèvres qui attrapent les aliments" doucement. Ensuite c'est un autre trait morphologique qui est mis en relief: "les torsades se détendaient" (Carême, 1976: 51). Le nez subit également une transformation: “[...] les replis de son nez s'étaient accentués. À la place des narines, deux fentes minces s'ouvraient et se refermaient à la cadence d'un mouvement respiratoire" (Carême, 1976: 63). Le processus physique s'arrête pour donner lieu à la mise en discours du regard qui porte déjà un attribut humain. La méduse communiquait toute intéressée aux productions culturelles telles que la peinture, le regard fixé au tableau de Rembrandt accroché au mur, la musique, et par hypothèse déductive, la poésie.

Or, si la métamorphose domine la dénaturation de l'animal, elle ne sera pas achevée. L'être n'atteindra pas la stabilité attendue, et la transformation, au lieu d'aboutir, sera, au contraire, réversible. Les épisodes de dégradation et distorsion ne préparent pas l'aboutissement métamorphique, la dernière touche due à une rupture reste dans l'implicite. Le passage n'étant pas accompli tournera en régression produisant de l'inertie. En effet, "L'éclat de ses yeux s'éteint. Elle n'avait plus figure humaine. [...] De la tête, il ne restait qu'une masse de gélatine visqueuse" (Carême, 1976: 82).

Reprenons la figure de l'écrivain. Le jeu opéré dans la narration nous conduit à repérer d'abord les éléments annonciateurs: le narrateur-témoin cherche à lire dans les diverses rencontres qu'il traverse les signes de son destin d'écrivain. La figure de l'écrivain au travail dès le 4ème chapitre permet de laisser glisser entre les deux récits complémentaires des réflexions sur l'écriture afin d'augmenter l'authenticité de sa narration. Si les coulisses de l'écriture se révèlent avec une franchise, ce n'est pas anodin. Le fait de réfléchir à la production et à l'origine de l'écriture avec René Deharme met en jeu la perméabilité entre l'univers extradiégétique et l'univers diégétique. Et ce n'est pas tout. II y a la confrontation entre le Maurice Carême fictionnel, auteur d'un roman qu'il est incapable d'écrire, "étranger à lui-même" tout en racontant l'objet de cette écriture manquée, et Maurice Carême, auteur réel qui est en train d'effectuer un voyage comme envoyé pour un journal de Paris. 
Ce qui préoccupe le narrateur-écrivain, ce sont le caractère visible et le caractère indicible du réel. En tant que narrateur, il se donne à discourir de ses efforts qui consistent à rendre sa curieuse expérience visuelle par le moyen de l'écriture. Les effets de fiction dans la narration se produisent à travers sa croyance qu'il est doublement victime d'une illusion: il pense que l'histoire étrange qu'il relate est fictive alors qu'elle peut être véridique, et en même temps il croit qu'elle est vraie alors qu'elle peut être absolument inventée.

II se trouve finalement dans une situation paradoxale: si l'écriture comporte nécessairement une part de l'imagination, il échoue dans sa tentative initiale. S'il a de la peine à écrire ce n'est pas forcément parce que les images seraient évanescentes et échapperaient à toute fixation verbale. La raison pour laquelle l'écriture est vouée à l'échec consiste en ce qu'il cherche l'ordre à savoir l'enchaînement causal là où il n'y a pas. Les associations n'obéissent à aucune logique causale, elles naissent de la singularité de l'imaginaire propre à l'individu à l'instar des images oniriques, comme le montre son rêve bizarre qui n'arrête pas de recommencer. Les dispositions d'esprit de l'écrivain suivent la voie du rêve au sein duquel les associations, sous la dictature du désordre, n'interdisent pas l'assimilation entre les personnages qu'ils soient réels ou imaginaires: les Coréens qui dans l'état de veille du narrateur sont présentés dans le journal, ainsi complètement ancrés dans le référentiel, Médua et le narrateur apparaissent ensemble dans l'espace crée par le rêve pour faire apparaître une configuration inédite: "la tête de Médua tombait arrachée, près de la mienne" (Carême, 1976: 43).

Dans cet état de confrontation entre associations et causalités, le seul procédé pour donner lieu à la création correspond à des déclarations d'impuissance elles-mêmes. Elles ont pour fonction de signaler que l'image que le narrateur-écrivain se fait de l'événement qui demande à être mis en discours ne peut pas être équivalente à l'événement vécu. Du coup, il n'est pas étonnant que l'imagination en tant que support tende à nourrir les images produites par son esprit telles que rêves, anticipations, suggestions, hallucinations. Dû à cet échec, le narrateur-écrivain invite à partager son espace mental, et de ce fait, à entrer dans le jeu fictionnel en faveur de la fragilisation de la fonction représentationnelle.

En somme, le narrateur fait tout pour dissimuler le caractère fictif en ancrant le récit dans le référentiel. Les signes de la narration effacés au début donnent lieu à l'histoire qui se confond avec la réalité du fait que l'écrivain se met à réfléchir, au seuil de l'écriture, au caractère réel de ce qu'il vit et observe. Malgré cet effort, le narrateur ne peut pas éviter d'afficher le caractère fictif une fois qu'il a pris la posture de l'écrivain. 


\section{Les usages de la métamorphose: perspective auditive}

Dans le récit de Marcel Thiry, Nondum jam non l'espace, contrairement à ce que nous avons observé chez Carême, va se rétrécissant, et ceci, pour donner lieu à une ouverture subversive dans le temps. Le récit suggère une structure temporelle paradoxale qui se manifeste à travers l'encadrement spatial hermétique de la chambre, aménagée également en lieu d'attente, dans laquelle l'évocation des souvenirs est effectuée dans la permanence d'un rituel incantatoire. D'un côté, cette structure temporelle est bâtie sur une prophétie relevant d'un autre temps distinct de celui du narrateur-protagoniste, projection de l'avenir dans la mesure où les mugissements des chars des pompiers font advenir la figure de l'ombre. D'un autre côté, elle est bâtie en même temps sur les réminiscences relevant également d'un autre temps bien distinct du premier, reconstruction du passé dans la mesure où la figure de l'ombre l'invite à y intervenir afin d'effectuer des modifications. En effet, la mémoire involontaire, à cause de sa structure paradigmatique contribue à infléchir ou à faire basculer le cours des événements de manière à renverser la flèche du temps. Cependant, prophétie et réminiscences mettent en question la métamorphose qui anime et prend en charge cette vision du temps.

Le narrateur-protagoniste lors de son déplacement habituel entre la banque et son appartement est toujours fasciné par les effets sonores qui le frappent régulièrement et s'inscrivent définitivement dans son esprit. Le mode d'appréhension du monde pour lui ne consiste pas en l'acuité du regard comme c'est le cas pour le narrateur de Médua mais en l'acuité de l'ouï impliquant une évolution métamorphique qui affecte inéluctablement le temps. Comme le remarque Hallin-Bertin dans son étude ayant pour objectif principal d'aborder l'œuvre de Thiry dans l'optique du fantastique ${ }^{8}$, le récit est organisé autour d'une métaphore qui sera complètement absorbée par la réalité "et le récit réalise la métamorphose que propose la métaphore" (Hallin-Bertin, 1981: 79).

Grâce à ses capteurs sensibles que sont les oreilles, son monde est ainsi composé d'une multitude de voix dont les origines ne sont pas pourtant des sujets parlants dotés de la faculté du langage. Ce sont "les marteaux-pics et les machines à coudre, les trommels des bétonneuses et les roues des wagons" en somme, les mécaniques qui ne font pas tout simplement des bruits mais "disent des mots" (Thiry, 1981: 326) qu'il s'efforce à son tour de traduire, comme par exemple dans le cas de l'essuie-glace qui répétant Fête, nom de son amoureuse disparue, l'amène à chercher autre chose notamment Faute ou Pente. Le récit donne ainsi lieu à des moments exceptionnels de dialogisme en face des "choses qui parlent

\footnotetext{
${ }^{8}$ Par ailleurs, dans cette approche la poétisation du fantastique est étroitement liée aux "préoccupations métaphysiques qui traversent l'œuvre en prose de Marcel Thiry" et qui "se fondent sur une hantise morale essentielle: le sentiment de la faute" (Hallin-Bertin, 1981: 101). Notre approche centrée sur la narration peut se passer de considérations morales aussi bien que métaphysiques.
} 
pour leur compte, qui ne laissent pas domestiquer leur bruit, qui ne se laissent pas spolier de ce qu'elles ont à dire" (Thiry, 1981: 326). Par ailleurs, cette attitude dialogique est une condition préalable qui doit être satisfaite pour la réalisation de la métamorphose.

En incipit, le narrateur est dans la même position mentale et émotionnelle que celui de Médua dans la mesure où il lui incombe, à lui aussi, de recevoir des indices étranges, de substance cette fois-ci prioritairement sonore, qui viennent nettement de l'extérieur. II croit capter des paroles alors que ce ne sont que les signaux de la sirène. Un signal est une modification de l'environnement produit par un dispositif qui doit être traité par un autre dispositif pour qu'il y ait communication entre eux, et dans ce cas de figure, puisque ce sont les chars des pompiers, il devrait porter comme message l'avertissement d'un incendie. Or, le passage des chars qui sont privés de cet avertissement d'incendie prend un nouveau statut, celui d'une manifestation prémonitoire auquel donnent accès des comparaisons. En effet, un examen minutieux pourrait recenser les analogies qui sont loin d'être gratuites certes mais que nous préférons écarter car cela nous obligerait d'ouvrir une nouvelle piste à suivre.

Revenons donc aux signes sonores. Le fa mi, fa mi changé plus tard en mi ré, mi ré des sirènes des chars doit laisser le narrateur identifier des paroles en latin pour exclure le cas de figure où elles auraient pour origine ses hallucinations. Puisqu'il ne connaît pas le latin, cette "langue sacrée et prophétique", "de la profération pythique" (Thiry, 1981: 327), sert de preuve pour le caractère éminemment annonciateur des paroles dont la source n'est pas son imagination inventive mais, par déduction, "une personnalité souterraine" (Thiry, 1981: 328). Voilà la manière dont les paroles s'articulent pour faire déployer du sens:

oui, ce mugissement voulait, et il voulait dire - voulait dire un mot latin, le mot nondum; [...] je le reconnaissais subitement avec sa signification, pas encore [...] et brusquement, au moment exact où la voiture clamante perforait la frontière par son passage à ma hauteur, la clameur changeait de nature et de timbre, une modification instantanée en renversait la vapeur, et elle versait dans le passé à toute vitesse le fa mi, fa mi devenu mi ré, mi ré [...] et le nondum, je le découvris avec un grave émerveillement, changé en jam non, ou déjà plus, jamais plus (Thiry, 1981: 327).

II ne faudrait pas cependant oublier la dimension visuelle. Elle est aussi évoquée mais contrairement à la dimension auditive, elle est complètement détachée de l'extérieur et c'est pour servir à escamoter le déroulement même de la métamorphose: le narrateur voit des couleurs les yeux fermés. Ce n'est pas seulement la mémoire involontaire qui donne lieu à la métamorphose du temps sur la base des jeux de couleurs mais aussi cet espace imaginaire a priori invisible qui se crée sur la rétine intérieure et auquel il a recours à 
plusieurs reprises: "et à ce moment du coup de baguette inverseur, en même temps que le nondum culbutait en jam non, ma vue intérieure percevait le subit changement de couleur de la lancée, qui rejaillissait vers le rouge comme en remontée de plongeur" (Thiry, 1981: 328). Le changement est donc accessible grâce aux couleurs qui engagent l'altération du temps: "le futur propagé vers moi en trombe émergeait bien du rouge pour verser au violet, tandis que sur l'autre versant les jam non en fuyant retournaient au même rouge originaire et final" (Thiry, 1981: 332).

Pour mettre en valeur la temporalité, il est nécessaire de priver les chars de toute idée d'urgence. C'est à cette condition que les hurlements apparaissent comme un nouveau code où un message se trouverait à demander à son destinataire un éprouvant décryptage tel un oracle; c'est un langage qui par le biais de l'événement annoncé plonge dans le temps et nourrit le sentiment de l'inévitable, donc l'événement accompli. Les rapports entre l'oracle et le double ont été étudiés dans une perspective non narratologique mais philosophique par Rosset. Nous en retenons l'idée selon laquelle la structure oraculaire tient du double dans la mesure où l'événement attendu et l'événement réel se trouvent en relation de substitution entre eux de manière à susciter l'inattendu tout en gardant l'idée de la conformité: "L'événement attendu vient coïncider avec lui-même d'où précisément la surprise: car on attendait quelque chose de différent, quoique voisin, la même chose mais pas exactement de cette façon" (Rosset, 1984: 41).

Dans le prolongement de ces idées, le double de ce genre met en cause également l'écoulement du temps qu'il faudrait constater irréversible parce que linéaire, et ceci, surtout dans le suivi des actes. Or, la coïncidence et la conformité ouvrent une nouvelle dimension. C'est la mémoire qui permet de retourner en arrière. Contrairement au temps séquentiel et vectoriel des actions qui composent une histoire, le temps du souvenir qu'affectionne le protagoniste semble celui des émotions. Les chars et la figure de l'ombre constituent des supports pour la mémoire et lui permettent de revivre les événements du passé non pas sur une succession ordonnée. Dans cette perspective, la mémoire n'est pas un simple miroir des événements de la réalité du passé. Elle est une forme de connaissance qui est activée par des états émotionnels sous la dominance de la perception auditive. La figure de l'ombre permet de plonger dans le temps pour en remonter le temps renversé. Le caractère nonlinéaire de la mémoire met en cause toute chronologie et tout rapport de filiation. Sur la base des rapports étroits entre l'annonce et la reconstruction du passé, entre les événements et la mémoire, le passé et le futur seront intervertis: c'est le passé qui est mis en avant parce qu'il est sous les yeux du narrateur, et c'est le futur qui est mis en arrière parce qu'il est invisible.

Les sirènes qui montent de ma gauche disent Jam non et non plus nondum, c'est le passé qui vient vers nous, ce n'est pas le futur, et quand les chars passent juste à ma 
hauteur et que leur hourra baisse d'un demi-tour par brusque cassure et basculent dans l'éloignement, ce n'est plus jam non, c'est nondum qui va se perdre dans les faubourgs de ma droite, du côté où s'est évanouie la seize-chevaux. Le Temps est renversé (Thiry, 1981: 389-390).

Cette manipulation correspond parfaitement à ce que Hallin-Bertin appelle "poétique du comme si" fondée sur "l'effacement des frontières entre les champs d'expérience" (HallinBertin, 1981: 135).

Quant à l'énigme, nous décelons chez Thiry quelque chose de similaire au procédé mis en œuvre dans Médua. D'un côté, c'est le narrateur qui subit une transformation mais le processus de modification n'est pas décrit seulement pris en charge par lui, et d'un autre côté, c'est l'identité de l'ombre qui recèle en soi une énigme. Est-ce qu'elle est le résultat d'une métamorphose mise en scène en son stade d'aboutissement? Et si c'est le cas, quel était l'état initial? Si c'est le narrateur, alors malgré toute transformation, il ne peut se métamorphoser en un autre être que lui-même?

De toute manière, l'événement est appréhendé tantôt comme aboutissant à une transformation, tantôt comme stabilité exluant toute transformation. Le récit raconte la contradiction selon laquelle la création imaginaire est susceptible de créer un passé qui ne dépend plus du déroulement préalable. Dans cet espace construit qui se greffe sur celui de sa chambre, le dialogue avec la figure de l'ombre crée de la fiction au sein de laquelle il obtient une connaissance qui ne pourrait pas être acquise dans son existence factuelle. Son identité tient à l'événement qui le change, peu importe s'il est grave ou futile (Romano: 1998).

La logique de la métamorphose que le récit intègre dans son propre mouvement n'est pas tellement la logique de l'exception ou celle de la négativité. Elle consiste à transformer la métamorphose de l'événement en événement de la métamorphose, ce qui explique l'absence de la mise en scène des phases traversées lors du processus.

\section{En guise de conclusion}

Au terme de nos réflexions, nous rappelons l'enjeu de notre entreprise qui consiste à forger, pour les besoins de nos propos, un outil sur la base de la métamorphose grâce à laquelle il nous paraît possible de saisir la manière dont le caractère de construction de l'œuvre dans son intégralité tend à s'afficher et à faire basculer le réel diégétique vers la fiction sans sacrifier pour autant l'autorité narrative sur la base des jeux de brouillage. II devient aussi manifeste que la même attitude envers le réel et l'imaginaire constitue un obstacle à l'établissement d'une distinction nette entre ce qui est représenté et ce qui 
représente. La valeur heuristique de notre outil, sur la base des deux points de vue précédents, consiste à mettre au jour de nouveaux aspects relatifs à l'imaginaire indépendamment de toute question générique, en annonçant la nouvelle epistemé du monde contemporain.

En effet, ces récits contribuent à faire percevoir que le phénomène littéraire ne se ramène pas à la représentation mais est composé de manières de dire pour mettre en scène tout particulièrement ce qui mérite pour un narrateur d'être raconté, et ceci, par le biais de la métamorphose.

La métamorphose remet en question les frontières entre matière et esprit, réel et fictif, rendant justice ainsi à la richesse cognitive des œuvres de fiction.

D'un côté, il semblerait que la fiction ne doive pas être enfermée dans le référentiel imaginaire de son monde possible et qu'elle puisse au contraire fournir un moyen particulièrement fécond d'accéder au réel. $M$. Butor affirme ainsi le réalisme de la fiction. De l'autre, c'est la réalité tout entière qui apparaît comme une construction imaginaire, ou du moins c'est la manière dont nous l'appréhendons, et nous cherchons à la restituer par nos discours qui est critiquée et complexifiée (Baroni et Meier, 2007: 4).

Les textes développent de manière différente la mise ne scène et la mise en discours de la métamorphose mais qui, dans les deux cas fait l'objet d'un constat d'une instance narrative, sensible à ébranler le sentiment de réalité, en réhabilitant l'imagination. Dans ce contexte, tout concourt à miner le réel: au lieu de raconter un récit représentant des événements qui inciterait à son tour le lecteur à les imaginer, on nous raconte un récit qui représente qu'on imagine des événements. La posture du narrateur incite à imaginer de manière à suggérer que le caractère fictif soit susceptible de faire naître des représentations.

S'il est vrai que l'objet de la représentation est l'imagination, la visée référentielle n'est pas supprimée non plus. Le glissement entre fictif et réel tend à indiquer le fait qu'aucune priorité n'est donnée ni à l'un ni à l'autre. Si le déséquilibre n'a pas lieu, c'est parce que la distinction n'est pas d'ordre ontologique mais épistémologique. Ainsi, la prise en compte du statut de l'imaginaire au sein de la fiction n'éloigne pas le réel mais au contraire semble le rattraper.

Pour terminer, nous nous proposons d'indiquer deux pistes à explorer, puisque nous sommes consciente du fait que notre parcours sommaire soulève suffisamment de vastes problèmes qui ne pourraient pas être résolus dans le cadre de ce travail.

D'une part, nous avons laissé certaines questions ouvertes concernant les rapports que la métamorphose pourrait entretenir avec le fantastique. Nous n'avons fait que suggérer 
un rapport possible à savoir si la métamorphose est liée au fantastique c'est parce qu'en tant que procédé discursif elle donne lieu à l'énigmatique. Or, les questions de savoir si une entité soumise à un changement radical deviendrait, de ce fait, fantastique, ou encore que tout changement provoquerait un effet de fantastique, sont aussi légitimes.

D'autre part, les réflexions sur la métamorphose soulèvent des interrogations qui dépassent le cadre narratologique proprement dite: Est-ce que la fiction est un type spécifique de représentation ou un usage spécifique de représentations?

\section{Bibliographie}

ACHARD-BAYLE, Guy (2001). Grammaire des métamorphoses, référence, identité, changement, fiction, Bruxelles: Éditions Duculot.

AugÉ, Marc (1999). Pour une anthropologie des mondes contemporains. Paris: Flammarion. Coll. "Champs".

BARONI, Raphaël et MeIER, Daniel (2007). "Le monde, en discours et en fictions". In: $A$ contrario, vol ${ }^{\circ} 5, \mathrm{n}^{\circ} 1$, pp. 3-7.

BARONIAN, Jean-Baptiste (2000). Panorama de la littérature fantastique de langue française. Tournai: La Renaissance du Livre. Coll. "Les maîtres de l'imaginaire".

BERTHELOT, Francis (1993). La métamorphose généralisée, du poème mythologique à la science-fiction. Paris: Nathan.

BOUVET, Rachel (1998). Étranges récits, étranges lectures: essai sur l'effet fantastique. Québec: Presses de l'Université du Québec.

BRunEL, Pierre (2004). Le mythe de la métamorphose. Paris: José Corti.

CARÊME, Maurice (1976). Médua. Waterloo: La Renaissance du livre.

DAmBre, Marc et Gosselin-NoAT, Monique (dir.) (2001). L'éclatement des genres au XXe siècle. Paris: Presses de la Sorbonne Nouvelle.

DuBoIS, Claude-Gilbert dir. (1985). L'imaginaire du changement, conversion, modification, métamorphoses, tome II. Bordeaux: Presses universitaires de Bordeaux.

DuCROT, Oswald et SCHAEFFER, Jean-Marie (1995). Nouveau dictionnaire encyclopédique des sciences du langage. Paris: Éditions du Seuil. Coll. "Points essai", pp. 545-546.

FRONTISI-DUCROUX, Françoise (2009). "L'invention de la métamorphose". [en ligne] In: Rue Descartes, 2009/2 n64, pp. 8-22. [consulté le 13/02/2012]

<URL: http://www.cairn.info/revue-rue-descartes-2009-2-page-8.htm>.

HALLIN-BERTIN, Dominique (1981). Le fantastique dans l'œuvre en prose de Marcel Thiry. Bruxelles: Palais des Académies. 
LASCU-POP, Rodica (1994). "Maurice Carême dans l'orbite du fantastique". In: Itinéraires et contacts des cultures, La Littérature belge de langue française. Paris: éd. L'Harmattan, pp. 121-127.

LIPOVETSKY, Gilles (1983). L'ère du vide. Paris: Gallimard. Coll. "Folio essai".

LITS, Marc (1993). “Des fantastiqueurs belges?” In: Textyles, n¹0, pp. 7-23.

MARIGNY, Jean (1987). "Réflexion autour de la notion de fantastique". In: Les Cahiers du GERF, $\mathrm{n}^{\circ} 1$, pp. 7-20.

PIGEAUD, Jackie (1995). L'Art et le vivant. Paris: Gallimard.

QUAGHEBEUR, Marc (2012). "Le Voyage rétrospectif. Un texte important de Franz Hellens". In: Studi Francesi, anno LVI, fasc. I, n¹66, pp. 99-109 [en ligne]. Bruxelles: Archives et Musée de la littérature. [consulté le 12/03/2012] <URL: http://www.aml.cfwb.be>.

ROMANO, Claude (1998). L'événement et le monde. Paris: P.U.F.

Rosset, Clément (1984). Le réel et son double. Paris: Gallimard.

THIRY, Marcel ([1966] 1981). "Nondum jam non". In: Romans, nouvelles, contes, récits. Bruxelles: André de Rache, pp. 321-393.

TONNET-LACROIX, Éliane (1993). La littérature française dans l'entre-deux-guerres. Paris: Nathan. 